\title{
Complex-Analytic Approach to the Sinc-Gauss Sampling Formula
}

\author{
Ken'ichiro Tanaka, Masaaki Sugihara ${ }^{\dagger 1}$ and Kazuo Murota ${ }^{\dagger 2}$ \\ Department of Mathematical Informatics \\ Graduate School of Information Science and Technology \\ University of Tokyo, Tokyo 113-8656, Japan \\ ${ }^{\dagger 1}$ E-mail: m_sugihara@mist.i.u-tokyo.ac.jp \\ ${ }^{\dagger 2}$ E-mail: murota@mist.i.u-tokyo.ac.jp
}

Received April 20, 2007

Revised September 5, 2007

\begin{abstract}
This paper is concerned with theoretical error estimates for a sampling formula with the sinc-Gaussian kernel. Qian et al. have recently given an error estimate for the class of band-limited functions by Fourier-analytic approach. In contrast, we adopt in this paper a complex-analytic approach to derive an error estimate for a wider class of functions including unbounded functions on $\mathbf{R}$. Part of the result of Qian et al. can be derived from ours as an immediate corollary. Computational results show a fairly good agreement with our theoretical analysis.
\end{abstract}

Key words: sampling formula, sinc-Gaussian kernel, sinc numerical methods

\section{Introduction}

Numerical methods based on sinc approximation, i.e., "sinc numerical methods," have recently been enjoying increasing popularity [6], [7], [8]. The basic idea underlying this approach is to employ the sinc function,

$$
\operatorname{sinc}(x)=\left\{\begin{array}{cc}
\frac{\sin (\pi x)}{\pi x} & (x \neq 0) \\
1 & (x=0)
\end{array}\right.
$$

as the basis for function approximation of the form

$$
f(x) \approx \sum_{k=\lfloor x / h\rfloor-N}^{\lceil x / h\rceil+N} f(k h) \operatorname{sinc}(x / h-k) .
$$

This expansion is motivated, of course, by Shannon's sampling theorem [1], which states that the identity

$$
f(x)=\sum_{k=-\infty}^{\infty} f(k h) \operatorname{sinc}(x / h-k) \quad(x \in \mathbf{R})
$$

holds if $f$ is band-limited in the sense that the Fourier transform $\hat{f}(\omega)$ of $f$ vanishes for every $\omega \in \mathbf{R}$ with $|\omega|>\pi / h$. 
It is customary in "sinc numerical methods" that the basic formula (1.2) is not used as it is, but some preliminary transformation is applied to the variable $x$. The transformation, say, $x=\varphi(t)$ is chosen so that the transformed function $f(\varphi(t))$ may decrease rapidly at infinity. Then the following formula results:

$$
f(x) \approx \sum_{k=-N}^{N} f(\varphi(k h)) \operatorname{sinc}(t / h-k) \quad\left(t=\varphi^{-1}(x)\right) .
$$

The truncation error ${ }^{1}$ incurred in this finite approximation is bounded by $\sum_{|k|>N}|f(\varphi(k h))|$. If $f(\varphi(t))$ decreases rapidly at infinity, say, exponentially, the truncation error decreases exponentially with respect to the number of sampled points $2 N+1$.

As a variant of the above idea we could introduce rapidly decreasing kernel functions to diminish the truncation error. A typical formula under this category is

$$
f(x) \approx\left(\mathcal{T}_{N, h} f\right)(x):=\sum_{k=\lfloor x / h\rfloor-N}^{\lceil x / h\rceil+N} f(k h) \operatorname{sinc}(x / h-k) \exp \left[-\frac{(x-k h)^{2}}{2 r^{2} h^{2}}\right],
$$

where $r$ is a positive constant, and $\lceil x\rceil$ denotes the least integer greater than or equal to $x$, and $\lfloor x\rfloor$ denotes the greatest integer less than or equal to $x$. Seeing that no standard name of this formula is found in the literature, we call this formula the sinc-Gauss sampling formula. This formula is used by Wei et al. in numerical solution of partial differential equations [11], [12], [13], [14], [15]. Qian et al. show that the error $\left\|f-\mathcal{T}_{N, h} f\right\|_{\infty}$ of the sinc-Gauss sampling formula decreases exponentially with respect to $N$ for a band-limited function $f$ and also demonstrate similar results about the approximation of the derivatives of $f[2],[3],[4],[5]$. In estimating the discretization error, they make use of the Fourier transform and the Parseval identity to exploit the band-limited condition. In Japan, as early as in 1975, H. Takahasi [9] proposed the sinc-Gauss sampling formula above and made an error analysis for holomorphic functions by using complex analysis. His analysis lacks, however, in mathematical rigor, although it captures the essential feature.

The objective of this paper is to provide a mathematically rigorous version of Takahasi's error analysis for the sinc-Gauss sampling formula. Furthermore, we point out that the formula is applicable to a wider class of functions including unbounded functions on $\mathbf{R}$. Specifically, we give an error estimate for those functions which are holomorphic on a band-shaped region on the complex plane

$$
\mathcal{D}_{d}:=\{z \in \mathbf{C}|| \operatorname{Im} z \mid \leq d\}
$$

and satisfy

$$
|f(z)| \leq A+B|z|^{\alpha} \quad\left(\forall z \in \mathcal{D}_{d}\right),
$$

\footnotetext{
${ }^{1}$ In general, the truncation error means the discrepancy between a finite sum $\sum_{-N}^{N}$ (as in (1.4)) and its limit $\sum_{-\infty}^{\infty}$. In contrast, the discretization error means the discrepancy between $f(x)$ and the approximation by the infinite sum $\sum_{-\infty}^{\infty}$.
} 
where $d>0, A \geq 0, B \geq 0$, and $\alpha \geq 0$. Furthermore, we show that part of Qian et al.'s result for band-limited functions can be derived from ours as an immediate corollary. It is mentioned that the error estimate for bounded functions (i.e., for the special case with $B=0$ ) has been discussed in [10].

The organization of this paper is as follows. In Section 2, we present our main results. In Section 3, we specialize our results to bounded functions, and explain the relationship to some results of Qian-Ogawa [5]. In Section 4 we show computational results. Proofs of theorems and lemmas are given in Section 5.

\section{Main results}

For nonnegative integer $m$ and positive numbers $r, h>0$, we define operators $\mathcal{G}_{h}^{(m)}, \mathcal{T}_{N, h}^{(m)}$ approximating the $m$-th order derivative $f^{(m)}$ of a function $f$ as

$$
\begin{aligned}
\left(\mathcal{G}_{h}^{(m)} f\right)(x) & :=\sum_{k=-\infty}^{\infty} f(k h) \frac{\mathrm{d}^{m}}{\mathrm{~d} x^{m}}\left[\operatorname{sinc}(x / h-k) \exp \left[-\frac{(x-k h)^{2}}{2 r^{2} h^{2}}\right]\right], \\
\left(\mathcal{T}_{N, h}^{(m)} f\right)(x) & :=\sum_{k=\lfloor x / h\rfloor-N}^{\lceil x / h\rceil+N} f(k h) \frac{\mathrm{d}^{m}}{\mathrm{~d} x^{m}}\left[\operatorname{sinc}(x / h-k) \exp \left[-\frac{(x-k h)^{2}}{2 r^{2} h^{2}}\right]\right],
\end{aligned}
$$

where sinc is the function defined in (1.1). Note that (2.2) with $m=0$ coincides with (1.5). We call the formula given by $\mathcal{T}_{N, h}^{(m)} f$ the sinc-Gauss sampling formula.

Let $\mathcal{D}_{d}$ be the band-shaped region defined in (1.6). In this section, we assume that $f: \mathcal{D}_{d} \rightarrow \mathbf{C}$ is a holomorphic function on $\mathcal{D}_{d}$ with $|f(z)| \leq A+B|z|^{\alpha}\left(\forall z \in \mathcal{D}_{d}\right)$ for constants $A \geq 0, B \geq 0$ and $\alpha \geq 0$. The error of the formula will be measured by the supremum of the absolute value of $f(x)-\left(\mathcal{T}_{N, h}^{(m)} f\right)(x)$ over a finite interval $[-L, L]$ for $L>0$. The proofs of Lemma 2.1 and Lemma 2.2 below are given in Section 5.1 and Section 5.2, respectively.

First, the discretization error of the sinc-Gauss sampling formula is estimated as follows. In what follows $\mathbf{Z}_{+}$denotes the set of nonnegative integers.

Lemma 2.1 (Discretization error). Let $d>0$. Let $f: \mathcal{D}_{d} \rightarrow \mathbf{C}$ be a holomorphic function on $\mathcal{D}_{d}$ with $|f(z)| \leq A+B|z|^{\alpha}\left(\forall z \in \mathcal{D}_{d}\right)$ for constants $A \geq 0$, $B \geq 0$ and $\alpha \geq 0$. Let $m \in \mathbf{Z}_{+}, L>0, r>0$, and $h>0$ with $h \leq \min \{2 \pi d / \log 2, \pi\}$. Then we have

$$
\begin{aligned}
\sup _{-L \leq x \leq L}\left|f^{(m)}(x)-\left(\mathcal{G}_{h}^{(m)} f\right)(x)\right| & \\
\leq C_{0} \exp \left(-\frac{\pi d}{h}+\frac{d^{2}}{2 r^{2} h^{2}}\right)[ & C_{1} C_{3} \sqrt{2 \pi}+C_{2} C_{3} 2^{\frac{\alpha+1}{2}} \Gamma\left(\frac{\alpha+1}{2}\right) \\
& \left.+C_{1} 2^{\frac{2 m+1}{2}} \Gamma\left(\frac{m+1}{2}\right)+C_{2} 2^{\frac{\alpha+2 m+1}{2}} \Gamma\left(\frac{\alpha+m+1}{2}\right)\right],
\end{aligned}
$$

where

$$
C_{0}=\frac{2 \pi^{m-1}(m+3) ! r}{h^{m-1}}\left(1+\left(\frac{\sqrt{2}}{r h}\right)^{m}\right)\left(\frac{1}{d}+\frac{1}{d^{m+1}}\right)
$$




$$
\begin{aligned}
& C_{1}=A+2^{\alpha} B(L+d)^{\alpha}, \\
& C_{2}=2^{\alpha} B(r h)^{\alpha}, \\
& C_{3}=2+\left(\frac{\sqrt{2} d}{r h}\right)^{m} .
\end{aligned}
$$

Second, the truncation error of the sinc-Gauss sampling formula is estimated as follows.

Lemma 2.2 (Truncation error). Let $d>0$. Let $f: \mathcal{D}_{d} \rightarrow \mathbf{C}$ be a holomorphic function on $\mathcal{D}_{d}$ with $|f(z)| \leq A+B|z|^{\alpha}\left(\forall z \in \mathcal{D}_{d}\right)$ for constants $A \geq 0, B \geq 0$ and $\alpha \geq 0$. Let $m \in \mathbf{Z}_{+}, L>0, r>0$, and $h>0$. If $N \geq \max \{2, m r / \sqrt{2}, \sqrt{\lceil\alpha\rceil} r+1\}$, we have

$$
\sup _{-L \leq x \leq L}\left|\left(\mathcal{G}_{h}^{(m)} f\right)(x)-\left(\mathcal{T}_{N, h}^{(m)} f\right)(x)\right| \leq C_{0}^{\prime}\left(C_{1}^{\prime}+C_{2}^{\prime}\right) \exp \left[-\frac{(N-1)^{2}}{2 r^{2}}\right],
$$

where

$$
\begin{aligned}
C_{0}^{\prime} & =\frac{2 m ! \mathrm{e}^{\pi} \mathrm{e}^{\frac{3}{2 r^{2}}} r^{2}}{N(N-1) h^{m} \pi}, \\
C_{1}^{\prime} & =A+2^{\alpha} B\left[(L+h)^{\alpha}+2^{\alpha} h^{\alpha}\right], \\
C_{2}^{\prime} & =2^{2 \alpha} B h^{\alpha}(\lceil\alpha\rceil+1) ! ! \max \left\{(N-1)^{\lceil\alpha\rceil}, r^{\lceil\alpha\rceil}\right\} .
\end{aligned}
$$

From the lemmas above, we can derive the following error estimate by setting $h$ and $r$ appropriately for a given $N$.

Theorem 2.3 (Error of the sinc-Gauss sampling formula). Let $d>0$. Let $f: \mathcal{D}_{d} \rightarrow \mathbf{C}$ be a holomorphic function on $\mathcal{D}_{d}$ with $|f(z)| \leq A+B|z|^{\alpha} \quad\left(\forall z \in \mathcal{D}_{d}\right)$ for constants $A \geq 0, B \geq 0$ and $\alpha \geq 0$. Let $m \in \mathbf{Z}_{+}$and $L>0$. For a positive integer $N$, define $h$ and $r$ as

$$
h=\frac{d^{\prime}}{N}, \quad r=\sqrt{\frac{N}{\pi}}
$$

with an arbitrary constant $d^{\prime}$ satisfying $0<d^{\prime} \leq d$. Then we have

$$
\begin{aligned}
& \sup _{-L \leq x \leq L}\left|f^{(m)}(x)-\left(\mathcal{T}_{N, h}^{(m)} f\right)(x)\right| \\
& =\mathrm{O}\left(N^{2 m-\min \{1 / 2,1-\lceil\alpha\rceil+\alpha\}} \exp \left(-\frac{\pi N}{2}\right)\right) \quad(N \rightarrow \infty) .
\end{aligned}
$$

Proof. If $N$ is sufficiently large, the assumptions in Lemmas 2.1 and 2.2,

$$
h \leq \min \{2 \pi d / \log 2, \pi\}, \quad N \geq \max \{2, m r / \sqrt{2}, \sqrt{\lceil\alpha\rceil} r+1\}
$$


are satisfied under (2.10). We apply the lemmas to the right hand side of the inequality

$$
\begin{aligned}
& \sup _{-L \leq x \leq L}\left|f^{(m)}(x)-\left(\mathcal{T}_{N, h}^{(m)} f\right)(x)\right| \\
& \leq \sup _{-L \leq x \leq L}\left|f^{(m)}(x)-\left(\mathcal{G}_{h}^{(m)} f\right)(x)\right|+\sup _{-L \leq x \leq L}\left|\left(\mathcal{G}_{h}^{(m)} f\right)(x)-\left(\mathcal{T}_{N, h}^{(m)} f\right)(x)\right| .
\end{aligned}
$$

The estimate in Lemma 2.1 remains valid when $d$ is replaced by $d^{\prime}$. With $h$ and $r$ in $(2.10)$ we have

$$
\begin{aligned}
& \exp \left(-\frac{\pi d^{\prime}}{h}+\frac{d^{\prime 2}}{2 r^{2} h^{2}}\right)=\exp \left(-\frac{\pi N}{2}\right), \\
& \exp \left[-\frac{(N-1)^{2}}{2 r^{2}}\right]=\mathrm{O}\left(\exp \left(-\frac{\pi N}{2}\right)\right) \quad(N \rightarrow \infty) .
\end{aligned}
$$

Furthermore, the orders of $C_{0}, \ldots, C_{3}$ in Lemma 2.1 and $C_{0}^{\prime}, C_{1}^{\prime}, C_{2}^{\prime}$ in Lemma 2.2 as $N \rightarrow \infty$ are estimated as follows:

$$
\begin{aligned}
& C_{0}=\mathrm{O}\left(N^{\frac{3 m-1}{2}}\right), \quad C_{1}=\mathrm{O}(1), \quad C_{2}=\mathrm{O}\left(N^{-\frac{\alpha}{2}}\right), \quad C_{3}=\mathrm{O}\left(N^{\frac{m}{2}}\right), \\
& C_{0}^{\prime}=\mathrm{O}\left(N^{m-1}\right), \quad C_{1}^{\prime}=\mathrm{O}(1), \quad C_{2}^{\prime}=\mathrm{O}\left(N^{\lceil\alpha\rceil-\alpha}\right) .
\end{aligned}
$$

Thus we obtain the claim of the theorem.

The error estimate in Theorem 2.3 presupposes approximation of $f(x)$ at a single point $x$ and, accordingly, expresses the error bound in terms of the number $2 N+1$ of the sampling points required for a single point. In some situations, however, it is more natural to consider approximation over a finite interval $[-L, L]$ with $L>0$. This is the case, for instance, in applications to differential equations. In such a case it is more appropriate to express the error bound in terms of the number

$$
M=2\left(\frac{L}{d^{\prime}}+1\right) N
$$

of the sampling points needed for the approximation over the entire interval, rather than at a single point, where $d^{\prime}$ is in (2.10). In accordance with this, Theorem 2.3 can be recast into the following form.

Corollary 2.4. Let $d>0$. Let $f: \mathcal{D}_{d} \rightarrow \mathbf{C}$ be a holomorphic function on $\mathcal{D}_{d}$ with $|f(z)| \leq A+B|z|^{\alpha}\left(\forall z \in \mathcal{D}_{d}\right)$ for constants $A \geq 0, B \geq 0$ and $\alpha \geq 0$. Let $m \in \mathbf{Z}_{+}$and $L>0$. For a positive integer $N$, define $h$ and $r$ as (2.10), and $M$ as (2.11). Then we have

$$
\begin{aligned}
& \sup _{-L \leq x \leq L}\left|f^{(m)}(x)-\left(\mathcal{T}_{N, h}^{(m)} f\right)(x)\right| \\
& =\mathrm{O}\left(M^{2 m-\min \{1 / 2,1-\lceil\alpha\rceil+\alpha\}} \exp \left(-\frac{\pi d^{\prime}}{4\left(d^{\prime}+L\right)} M\right)\right) \quad(M \rightarrow \infty) .
\end{aligned}
$$




\section{Error estimates for bounded functions}

In this section, we present the error estimate for holomorphic functions $f: \mathcal{D}_{d} \rightarrow \mathbf{C}$ on $\mathcal{D}_{d}$ with $|f(z)| \leq A\left(\forall z \in \mathcal{D}_{d}\right)$ for a constant $A \geq 0$, and discuss its relationship to the estimate of Qian-Ogawa [5]. For bounded functions it is possible to consider supremum error bounds over the entire real number $\mathbf{R}$. The error estimates over $\mathbf{R}$ can be obtained easily from our results in Section 2 by setting $B=0, \alpha=0$ and letting $L \rightarrow \infty$. We set $\|g\|_{\infty}:=\sup _{-\infty<x<\infty}|g(x)|$ for a function $g$ on $\mathbf{R}$.

\subsection{Error estimates}

Letting $B=0, \alpha=0, L \rightarrow \infty$ in Section 2, we obtain the following lemmas and theorem shown in [10].

Lemma 3.1 ([10]). Let $d>0$. Let $f: \mathcal{D}_{d} \rightarrow \mathbf{C}$ be a holomorphic function on $\mathcal{D}_{d}$ with $|f(z)| \leq A\left(\forall z \in \mathcal{D}_{d}\right)$ for a constant $A \geq 0$. Let $m \in \mathbf{Z}_{+}, r>0$, and $h>0$ with $h \leq \min \{2 \pi d / \log 2, \pi\}$. Then we have

$$
\begin{aligned}
\left\|f^{(m)}-\mathcal{G}_{h}^{(m)} f\right\|_{\infty} \leq & \exp \left(-\frac{\pi d}{h}+\frac{d^{2}}{2 r^{2} h^{2}}\right) \\
& \cdot A\left[\frac{2 \pi^{m-1}(m+3) ! r}{h^{m-1}}\left(1+\left(\frac{\sqrt{2}}{r h}\right)^{m}\right)\left(\frac{1}{d}+\frac{1}{d^{m+1}}\right)\right] \\
& \cdot\left[\sqrt{2 \pi}\left(2+\left(\frac{\sqrt{2} d}{r h}\right)^{m}\right)+2^{\frac{2 m+1}{2}} \Gamma\left(\frac{m+1}{2}\right)\right] .
\end{aligned}
$$

Lemma 3.2 ([10]). Let $d>0$. Let $f: \mathcal{D}_{d} \rightarrow \mathbf{C}$ be a holomorphic function on $\mathcal{D}_{d}$ with $|f(z)| \leq A\left(\forall z \in \mathcal{D}_{d}\right)$ for a constant $A \geq 0$. Let $m \in \mathbf{Z}_{+}, r>0$, and $h>0$. If $N \geq \max \{2, m r / \sqrt{2}\}$, we have

$$
\left\|\mathcal{G}_{h}^{(m)} f-\mathcal{T}_{N, h}^{(m)} f\right\|_{\infty} \leq \frac{2 A m ! \mathrm{e}^{\pi} \mathrm{e}^{\frac{3}{2 r^{2}}} r^{2}}{N(N-1) h^{m} \pi} \exp \left[-\frac{(N-1)^{2}}{2 r^{2}}\right] .
$$

Theorem $3.3([10])$. Let $d>0$. Let $f: \mathcal{D}_{d} \rightarrow \mathbf{C}$ be a holomorphic function on $\mathcal{D}_{d}$ with $|f(z)| \leq A\left(\forall z \in \mathcal{D}_{d}\right)$ for a constant $A \geq 0$. Let $m \in \mathbf{Z}_{+}$. For $a$ positive integer $N$, define $h$ and $r$ as

$$
h=\frac{d^{\prime}}{N}, \quad r=\sqrt{\frac{N}{\pi}}
$$

with an arbitrary constant $d^{\prime}$ satisfying $0<d^{\prime} \leq d$. Then we have

$$
\left\|f^{(m)}-\mathcal{T}_{N, h}^{(m)} f\right\|_{\infty}=\mathrm{O}\left(N^{2 m-1 / 2} \exp \left(-\frac{\pi N}{2}\right)\right) \quad(N \rightarrow \infty) .
$$




\subsection{Relationship to Qian-Ogawa's result}

We investigate the relationship between the result of Qian-Ogawa [5] for bandlimited functions and our Theorem 3.3 in Section 3.1. For $\sigma>0$ let $B_{\sigma}$ be the set of band-limited functions defined as

$$
B_{\sigma}=\left\{f \in L^{2}(\mathbf{R})|| \omega \mid>\sigma \Rightarrow \hat{f}(\omega)=0\right\},
$$

where $\hat{f}$ denotes the Fourier transform of $f$.

The following theorem is immediate from Corollary 3.1 of [5].

Theorem 3.4 ([5]). Let $f \in B_{\sigma}$ and $0<h<\pi / \sigma$. For $N>2$, define $r=$ $\sqrt{(N-2) /(\pi-h \sigma)}$. Then we have

$$
\left\|f^{(m)}-\mathcal{T}_{N, h}^{(m)} f\right\|_{\infty}=\mathrm{O}\left(\frac{1}{\sqrt{N-2}} \exp \left[-\frac{(\pi-h \sigma)(N-2)}{2}\right]\right) \quad(N \rightarrow \infty) .
$$

The objective of this section is to demonstrate how (3.3) with $m=0$ can be derived from our result of Section 3.1. In the case of $m \geq 1$ we also derive a weaker result $^{2}$

$$
\left\|f^{(m)}-\mathcal{T}_{N, h}^{(m)} f\right\|_{\infty}=\mathrm{O}\left((N-2)^{(m-1) / 2} \exp \left[-\frac{(\pi-h \sigma)(N-2)}{2}\right]\right) \quad(N \rightarrow \infty) .
$$

First we note the following fact, which may be regarded as a part of the PaleyWiener theorem.

Lemma 3.5. If $f \in B_{\sigma}$, then $f$ is holomorphic on $\mathbf{C}$ and there exists a constant $A^{\prime} \geq 0$ such that

$$
|f(z)| \leq A^{\prime} \exp (\sigma|\operatorname{Im} z|) \quad(z \in \mathbf{C}) .
$$

Proof. Denote the Fourier transform of $f$ by $\hat{f}$. By $f \in B_{\sigma}$, we have $\hat{f} \in$ $L^{2}([-\sigma, \sigma])$ and

$$
f(z)=\frac{1}{\sqrt{2 \pi}} \int_{-\sigma}^{\sigma} \hat{f}(\omega) \exp (\mathrm{i} z \omega) \mathrm{d} \omega .
$$

Since the interval of integration is finite, we can exchange the differentiation and integration. Therefore $f$ is holomorphic on $\mathbf{C}$.

Next, again by (3.6), noting that $\hat{f} \in L^{2}([-\sigma, \sigma]) \subset L^{1}([-\sigma, \sigma])$, we have

$$
\begin{aligned}
|f(\xi+\mathrm{i} \eta)| & \leq \frac{1}{\sqrt{2 \pi}} \int_{-\sigma}^{\sigma}|\hat{f}(\omega)| \exp (\mathrm{i}(\xi+\mathrm{i} \eta) \omega) \mid \mathrm{d} \omega \\
& =\frac{1}{\sqrt{2 \pi}} \int_{-\sigma}^{\sigma}|\hat{f}(\omega)| \exp (-\eta \omega) \mathrm{d} \omega \\
& \leq \exp (|\eta| \sigma) \cdot \frac{1}{\sqrt{2 \pi}} \int_{-\sigma}^{\sigma}|\hat{f}(\omega)| \mathrm{d} \omega
\end{aligned}
$$

for $\xi, \eta \in \mathbf{R}$.

\footnotetext{
${ }^{2}$ The estimate (3.3) with $m \geq 1$ does not seem to be derivable from our results. This is because our estimate of the discretization error is considered under a more general condition, and is necessarily weaker.
} 
This lemma implies that our function class contains band-limited functions, stated in the following lemma.

Lemma 3.6. Let $f \in B_{\sigma}$. For any $d>0, f: \mathcal{D}_{d} \rightarrow \mathbf{C}$ is a holomorphic function on $\mathcal{D}_{d}$ with $|f(z)| \leq A\left(\forall z \in \mathcal{D}_{d}\right)$, where

$$
A=A^{\prime} \exp (\sigma d)
$$

with $A^{\prime}$ in (3.5).

This lemma enables us to apply Lemmas 3.1 and 3.2 to $f \in B_{\sigma}$. We take $r$ as in Theorem 3.4 and assume that $N$ is sufficiently large.

To estimate the discretization error, we set

$$
d=h(N-2)
$$

and take $A$ as (3.7). Then, by Lemma 3.1, we have

$$
\begin{aligned}
\left\|f^{(m)}-\mathcal{G}_{h}^{(m)} f\right\|_{\infty} \leq & A^{\prime} \exp \left(\sigma d-\frac{\pi d}{h}+\frac{d^{2}}{2 r^{2} h^{2}}\right) \\
& \cdot\left[\frac{2 \pi^{m-1}(m+3) ! r}{h^{m-1}}\left(1+\left(\frac{\sqrt{2}}{r h}\right)^{m}\right)\left(\frac{1}{d}+\frac{1}{d^{m+1}}\right)\right] \\
& \cdot\left[\sqrt{2 \pi}\left(2+\left(\frac{\sqrt{2} d}{r h}\right)^{m}\right)+2^{\frac{2 m+1}{2}} \Gamma\left(\frac{m+1}{2}\right)\right] .
\end{aligned}
$$

The degree of the underlined part with respect to $N-2$ is $(m-1) / 2$. The exponent of the remaining part is

$$
\begin{aligned}
\sigma d-\frac{\pi d}{h}+\frac{d^{2}}{2 r^{2} h^{2}} & =-\frac{(\pi-h \sigma) d}{h}+\frac{d^{2}}{2 r^{2} h^{2}} \\
& =-(\pi-h \sigma)(N-2)+\frac{(\pi-h \sigma) h^{2}(N-2)^{2}}{2(N-2) h^{2}} \\
& =-\frac{(\pi-h \sigma)(N-2)}{2} .
\end{aligned}
$$

Thus we obtain the following estimate:

$$
\left\|f^{(m)}-\mathcal{G}_{h}^{(m)} f\right\|_{\infty}=\mathrm{O}\left((N-2)^{(m-1) / 2} \exp \left[-\frac{(\pi-h \sigma)(N-2)}{2}\right]\right) \quad(N \rightarrow \infty) .
$$

To estimate the truncation error, we set $d=1$ and $A=A^{\prime} \mathrm{e}^{\sigma}$ according to (3.7). Then, by Lemma 3.2, we have

$$
\begin{aligned}
\left\|\mathcal{G}_{h}^{(m)} f-\mathcal{T}_{N, h}^{(m)} f\right\|_{\infty} & \leq \frac{2 A^{\prime} \mathrm{e}^{\sigma} m ! \mathrm{e}^{\pi} \mathrm{e}^{\frac{3}{2 r^{2}}} r^{2}}{N(N-1) h^{m} \pi} \exp \left[-\frac{(N-1)^{2}}{2 r^{2}}\right] \\
& \leq \frac{2 A^{\prime} \mathrm{e}^{\sigma} m ! \mathrm{e}^{\pi} \mathrm{e}^{\frac{3}{2 r^{2}}}}{h^{m} \pi} \frac{r^{2}}{(N-2)^{2}} \exp \left[-\frac{(N-2)^{2}}{2 r^{2}}\right] .
\end{aligned}
$$


The degree of $r^{2} /(N-2)^{2}$ with respect to $N-2$ is -1 . Furthermore, $\mathrm{e}^{\frac{3}{2 r^{2}}} \rightarrow 1$ as $N \rightarrow \infty$. The exponent of the remaining part is

$$
-\frac{(N-2)^{2}}{2 r^{2}}=-\frac{(\pi-h \sigma)(N-2)}{2} .
$$

Thus we obtain the following estimate:

$$
\left\|\mathcal{G}_{h}^{(m)} f-\mathcal{T}_{N, h}^{(m)} f\right\|_{\infty}=\mathrm{O}\left(\frac{1}{N-2} \exp \left[-\frac{(\pi-h \sigma)(N-2)}{2}\right]\right) \quad(N \rightarrow \infty) .
$$

By (3.8) and (3.9), we have (3.4) in the case of $m \geq 0$ and (3.3) in the case of $m=0$.

\section{Numerical experiments}

In this section, we present computational results on sinc-Gauss sampling formula for two types of functions: (i) rational functions

$$
f_{\beta, d}(z)=\frac{z^{\beta+2}}{z^{2}+d^{2}}
$$

with $\beta \in\{-2,-1,0,1,2\}$ and $d>0$, and (ii) band-limited functions

$$
f_{l}(z)=(\operatorname{sinc}(z))^{l}
$$

with a positive integer $l$. The former is not band-limited, and the latter is included to confirm that the performance of the sinc-Gauss sampling formula is essentially independent of the band-limited property of the functions to be approximated.

We consider errors on a finite interval $[-3,3]$ (i.e., $L=3$ ), which we evaluate numerically as the maximum of the errors at 6000 equally-spaced points in the interval. The relationship of the error against the number of sampling points will be presented in graphs. Specifically, the ordinates are the errors in logarithm,

$$
\log _{10}\left(\sup _{-3 \leq x \leq 3}\left|f(x)-\left(\mathcal{T}_{N, h}^{(m)} f\right)(x)\right|\right),
$$

and the abscissae are $N$ as well as $M=2\left(3 / d^{\prime}+1\right) N$ (with $L=3$ in (2.11)), where $M$ is indicated at the top.

According to our theoretical analysis summarized in Theorem 2.3, the error curves are expected to be almost linear, with the slope against $N$ being

$$
-\frac{\pi}{2} \log _{10} \mathrm{e}=-0.682 \cdots
$$

This theoretical slope will be compared with the observed values, which we obtain from the computational results by the least square method.

The program for the computation is written in C. Our computer is SUN Blade 2000 with the following environment: the operating system is Solaris 9, the CPU is UltraSPARC-III+ $(900 \mathrm{MHz}, 64$ bit) with $3 \mathrm{~GB}$ memory, the compiler is Sun Studio 11, in which "long double" is 128 bits wide. 


\subsection{Rational functions}

For $\beta \in\{-2,-1,0,1,2\}$ and $d>0$, define $f_{\beta, d}$ as

$$
f_{\beta, d}(z)=\frac{z^{\beta+2}}{z^{2}+d^{2}} \quad(z \in \mathbf{C}) .
$$

Then $f_{\beta, d}$ is holomorphic on $\mathcal{D}_{d-\varepsilon}$ for $\varepsilon$ with $0<\varepsilon \ll d$, and satisfies

$$
\left|f_{\beta, d}(z)\right| \leq \frac{\max \left\{d, d^{-1}\right\}}{\varepsilon}|z|^{\alpha} \quad\left(\forall z \in \mathcal{D}_{d-\varepsilon}\right),
$$

where $\alpha=\max \{\beta, 0\}$. The sinc-Gauss sampling formula $\mathcal{T}_{N, h}^{(m)}$ is applied to $f_{\beta, d}$ for $\beta=-2,-1,0,1,2, d=10^{-i}(i=0,1,2)$, and $m=0,1,2$. We set $\varepsilon=d / 100$ and $h=(d-\varepsilon) / N$. Furthermore, in computing the slopes, we exclude the data for $N=45$ and 50 to avoid the effect of rounding errors.

Table 1. $\log _{10}$ (max error) for $f_{\beta, d}$ of (4.3) with $\beta=-2$

\begin{tabular}{c||rr|rr|rr}
\multicolumn{1}{l||}{} & \multicolumn{2}{c|}{$m=0$} & \multicolumn{2}{c|}{$m=1$} & \multicolumn{2}{c}{$m=2$} \\
& $N=10$ & $N=40$ & $N=10$ & $N=40$ & $N=10$ & $N=40$ \\
\hline$d=1.00$ & -6.77 & -27.28 & -5.29 & -25.19 & -3.52 & -22.80 \\
$d=0.10$ & -4.77 & -25.32 & -2.29 & -22.20 & 0.48 & -18.84 \\
$d=0.01$ & -4.09 & -24.29 & 0.45 & -19.55 & 4.43 & -14.96
\end{tabular}

Table 2. $\log _{10}$ (max error) for $f_{\beta, d}$ of (4.3) with $\beta=-1$

\begin{tabular}{c||rr|rr|rr}
\multicolumn{1}{l||}{} & \multicolumn{2}{c|}{$m=0$} & \multicolumn{2}{c|}{$m=1$} & \multicolumn{2}{c}{$m=2$} \\
& $N=10$ & $N=40$ & $N=10$ & $N=40$ & $N=10$ & $N=40$ \\
\hline$d=1.00$ & -6.94 & -27.45 & -5.31 & -25.21 & -3.58 & -22.84 \\
$d=0.10$ & -5.96 & -26.47 & -3.31 & -23.21 & -0.59 & -19.84 \\
$d=0.01$ & -6.09 & -26.42 & -1.31 & -21.21 & 2.18 & -17.18
\end{tabular}

Table 3. $\log _{10}$ (max error) for $f_{\beta, d}$ of (4.3) with $\beta=0$

\begin{tabular}{c||rr|rr|rr}
\multicolumn{1}{c||}{} & \multicolumn{2}{c|}{$m=0$} & \multicolumn{2}{c|}{$m=1$} & \multicolumn{2}{c}{$m=2$} \\
& $N=10$ & $N=40$ & $N=10$ & $N=40$ & $N=10$ & $N=40$ \\
\hline$d=1.00$ & -6.89 & -27.35 & -5.40 & -25.26 & -3.65 & -22.87 \\
$d=0.10$ & -6.90 & -27.39 & -4.41 & -24.26 & -1.66 & -20.87 \\
$d=0.01$ & -7.38 & -28.14 & -3.53 & -23.52 & 0.34 & -18.87
\end{tabular}

Table 4. $\log _{10}$ (max error) for $f_{\beta, d}$ of (4.3) with $\beta=1$

\begin{tabular}{l||rr|rr|rr}
\multicolumn{1}{c||}{} & \multicolumn{2}{c|}{$m=0$} & \multicolumn{2}{c|}{$m=1$} & \multicolumn{2}{c}{$m=2$} \\
& $N=10$ & $N=40$ & $N=10$ & $N=40$ & $N=10$ & $N=40$ \\
\hline$d=1.00$ & -6.94 & -27.49 & -5.41 & -25.28 & -3.61 & -22.91 \\
$d=0.10$ & -6.91 & -27.66 & -4.41 & -24.56 & -1.60 & -21.15 \\
$d=0.01$ & -6.92 & -27.66 & -3.40 & -23.56 & 0.40 & -19.15
\end{tabular}



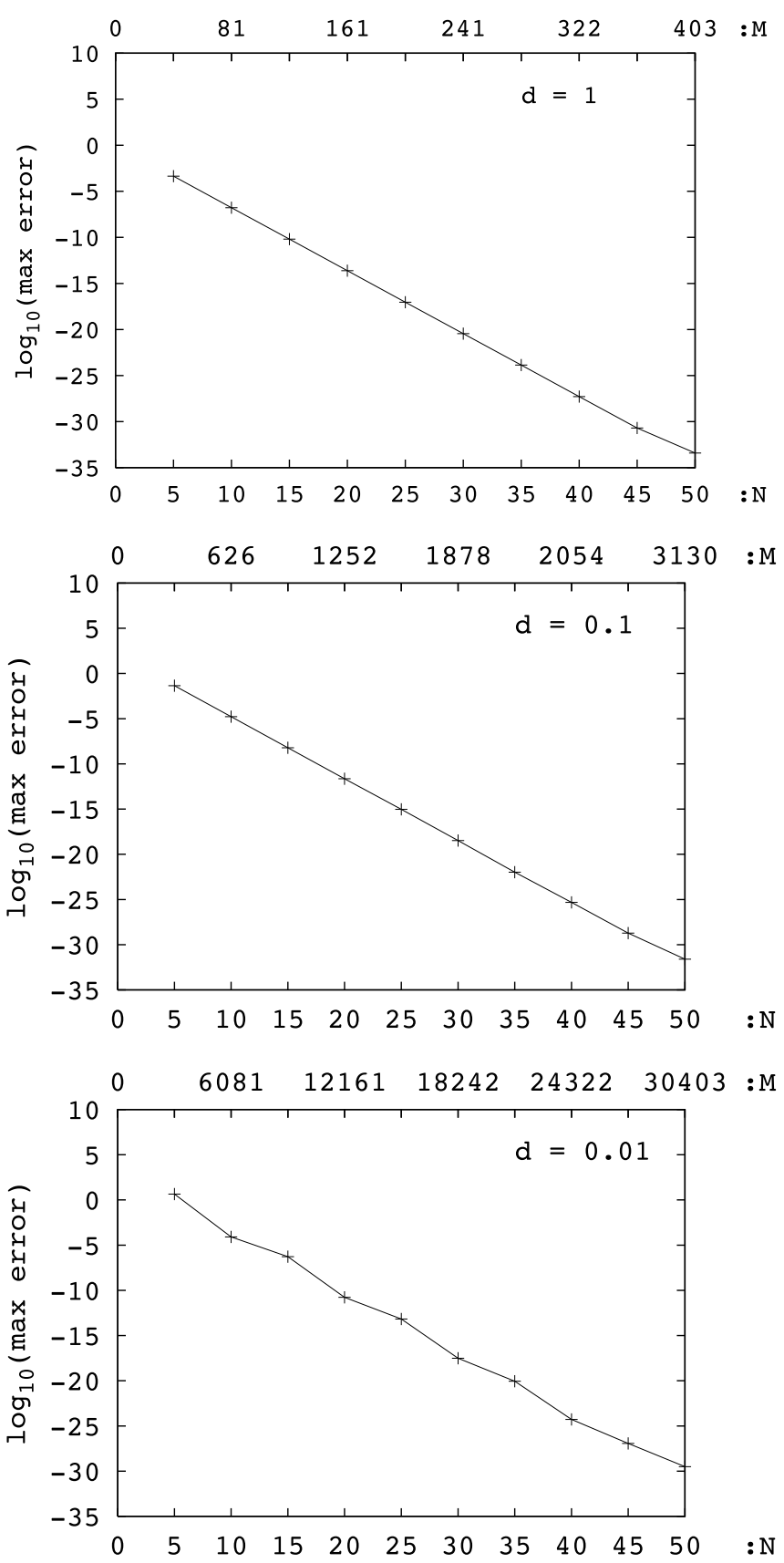

Fig. 1. Errors for $f_{\beta, d}$ of (4.3) with $\beta=-2$ and for $m=0$ 
Table 5. $\log _{10}$ (max error) for $f_{\beta, d}$ of (4.3) with $\beta=2$

\begin{tabular}{c||rr|rr|rr}
\multicolumn{1}{c||}{} & \multicolumn{2}{c|}{$m=0$} & \multicolumn{2}{c|}{$m=1$} & \multicolumn{2}{c}{$m=2$} \\
& $N=10$ & $N=40$ & $N=10$ & $N=40$ & $N=10$ & $N=40$ \\
\hline$d=1.00$ & -6.50 & -27.23 & -4.91 & -25.07 & -3.12 & -22.67 \\
$d=0.10$ & -6.43 & -27.19 & -3.93 & -24.08 & -1.13 & -20.68 \\
$d=0.01$ & -6.45 & -27.19 & -2.93 & -23.09 & 0.87 & -18.68
\end{tabular}

From Table 6-Table 10, we see that the experimental values of the slopes are close to the theoretical ones in (4.2). As $m$ (the order of differentiation) becomes larger, the slope tends to be larger than the theoretical value. This may be because we only use $\exp (-(\pi / 2) N)$ in Theorem 3.3 in computing the theoretical value, with the secondary factor $N^{2 m-\min \{1 / 2,1-\lceil\alpha\rceil+\alpha\}}$ disregarded.

Table 6. $\log _{10}$ (max error) $/ N$ for $f_{\beta, d}$ of (4.3) with $\beta=-2$

\begin{tabular}{l|rrr} 
& $m=0$ & $m=1$ & $m=2$ \\
\hline$d=1.00$ & -0.684 & -0.660 & -0.635 \\
$d=0.10$ & -0.686 & -0.660 & -0.636 \\
$d=0.01$ & -0.692 & -0.664 & -0.639
\end{tabular}

Table 7. $\log _{10}$ (max error) $/ N$ for $f_{\beta, d}$ of (4.3) with $\beta=-1$

\begin{tabular}{l|rrr} 
& $m=0$ & $m=1$ & $m=2$ \\
\hline$d=1.00$ & -0.684 & -0.660 & -0.634 \\
$d=0.10$ & -0.685 & -0.660 & -0.634 \\
$d=0.01$ & -0.691 & -0.660 & -0.638
\end{tabular}

Table 8. $\log _{10}$ (max error) $/ N$ for $f_{\beta, d}$ of (4.3) with $\beta=0$

\begin{tabular}{l|rrr} 
& $m=0$ & $m=1$ & $m=2$ \\
\hline$d=1.00$ & -0.681 & -0.658 & -0.632 \\
$d=0.10$ & -0.683 & -0.658 & -0.632 \\
$d=0.01$ & -0.694 & -0.663 & -0.632
\end{tabular}

Table 9. $\log _{10}$ (max error) $/ N$ for $f_{\beta, d}$ of (4.3) with $\beta=1$

\begin{tabular}{c|rrr} 
& $m=0$ & $m=1$ & $m=2$ \\
\hline$d=1.00$ & -0.687 & -0.660 & -0.637 \\
$d=0.10$ & -0.694 & -0.670 & -0.646 \\
$d=0.01$ & -0.694 & -0.670 & -0.646
\end{tabular}

Table 10. $\log _{10}$ (max error) $/ N$ for $f_{\beta, d}$ of (4.3) with $\beta=2$

\begin{tabular}{c|rrr} 
& $m=0$ & $m=1$ & $m=2$ \\
\hline$d=1.00$ & -0.693 & -0.670 & -0.646 \\
$d=0.10$ & -0.694 & -0.670 & -0.646 \\
$d=0.01$ & -0.693 & -0.669 & -0.646
\end{tabular}


Next, we consider the effect of $m$, the order of differentiation. By Theorem 3.3, we expect that $\log _{10}$ (max error) will increase approximately by $2 \log _{10} N$ if $m$ increases by one. The results of Table 6 -Table 10 agree with this expectation, whereas $m$ is also included in the constant part independent of $N$ in the estimate.

Next, we consider the effect of $d$, representing the location of the singular points. Noting the order with respect to $N$, we conclude that $d$ does not affect the error. It is expected, however, that $\log _{10}$ (max error) will increase approximately by $m+1$ if $d$ is multiplied by $1 / 10$, due to the term $1 / d^{m+1}$ in the estimate of Lemma 3.1. Computational results appear to support this observation.

Finally, we consider the effect of $\beta$. From the results, we see that $\beta$ does not affect the errors substantially, which is theoretically appropriate.

\subsection{Band-limited functions}

For a positive integer $l$, we define $f_{l}$ as

$$
f_{l}(z)=(\operatorname{sinc}(z))^{l} \quad(z \in \mathbf{C})
$$

Then we have $f_{l} \in B_{\pi l}$. The function $f_{l}$ is holomorphic on $\mathbf{C}$ and satisfies

$$
\left|f_{l}(z)\right| \leq \max \left\{\left(\frac{\mathrm{e}^{\pi d}}{\pi}\right)^{l}, \mathrm{e}^{\pi l}\right\} \quad\left(z \in \mathcal{D}_{d}\right)
$$

for arbitrary $d>0$. Setting $h=1 / N$, we apply the sinc-Gauss sampling formula to $f_{l}$ for $l=5,10,15,20$ and $m=0,1,2$. In computing the slopes, we exclude the data for $N=5,10,45,50$.

As to the effect of $m$ (the order of differentiation) on the errors, we see the same as in Section 4.1 .

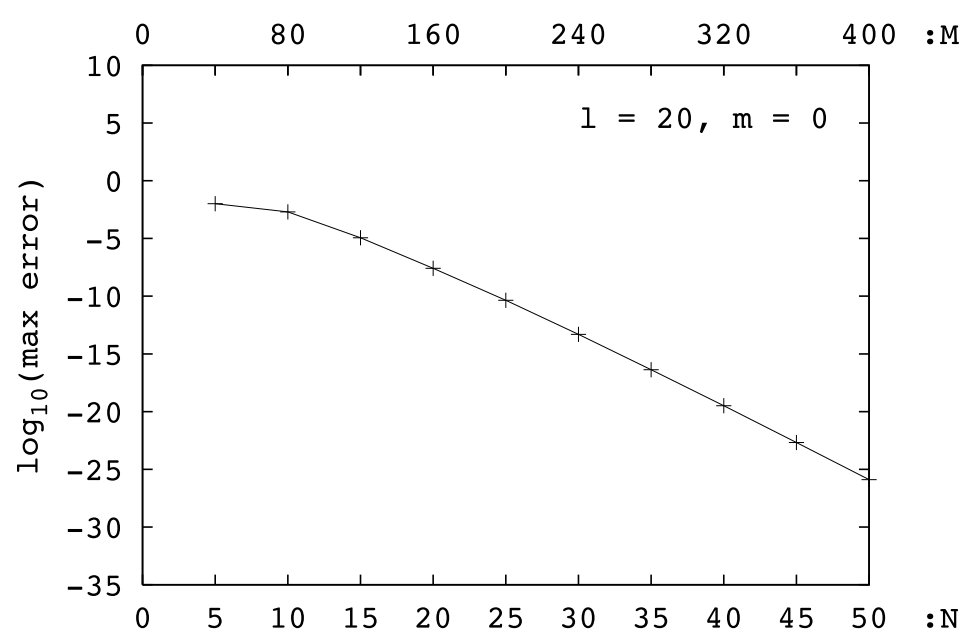

Fig. 2. Errors for $f=\operatorname{sinc}^{20}$ with $m=0$ and $d=1$ 
Table 11. $\log _{10}$ (max error) for $f=\operatorname{sinc}^{l}$ with $d=1$

\begin{tabular}{l||rr|rr|rr}
\multicolumn{1}{c||}{} & \multicolumn{2}{c|}{$m=0$} & \multicolumn{2}{c|}{$m=1$} & \multicolumn{2}{c}{$m=2$} \\
& $N=10$ & $N=40$ & $N=10$ & $N=40$ & $N=10$ & $N=40$ \\
\hline$l=5$ & -5.18 & -25.48 & -3.67 & -23.39 & -1.98 & -21.01 \\
$l=10$ & -3.99 & -23.18 & -2.39 & -21.09 & -0.81 & -18.74 \\
$l=15$ & -3.18 & -21.20 & -1.66 & -19.09 & -0.11 & -16.78 \\
$l=20$ & -2.70 & -19.49 & -1.23 & -17.38 & 0.34 & -15.09
\end{tabular}

Table 12. $\log _{10}$ (max error) $/ N$ for $f=\operatorname{sinc}^{l}$ with $d=1$

\begin{tabular}{l|rrr} 
& $m=0$ & $m=1$ & $m=2$ \\
\hline$l=5$ & -0.680 & -0.664 & -0.645 \\
$l=10$ & -0.653 & -0.638 & -0.618 \\
$l=15$ & -0.618 & -0.605 & -0.583 \\
$l=20$ & -0.583 & -0.568 & -0.546
\end{tabular}

Next, we consider the slopes of the error curves in the graphs. The experimental values of the slopes are close to the theoretical ones in (4.2) when $l$ is small (Table 12). In the case where $l$ is large, however, this is not the case. This may be because the constant on the right hand side of (4.5) is large when $l$ is large (note that $d=1)$, and the effect of the constant cannot be ignored.

Taking this fact into consideration, we apply the formula in the case of $d=$ $\pi^{-1} \log \pi$, i.e., $h=(\pi N)^{-1} \log \pi$. The results of the experiments are presented in Fig. 3 and Table 14, which justify the above observation. The numerical results support our expectation that smaller width between neighboring sampling points yields better approximation for functions with strong vibration.

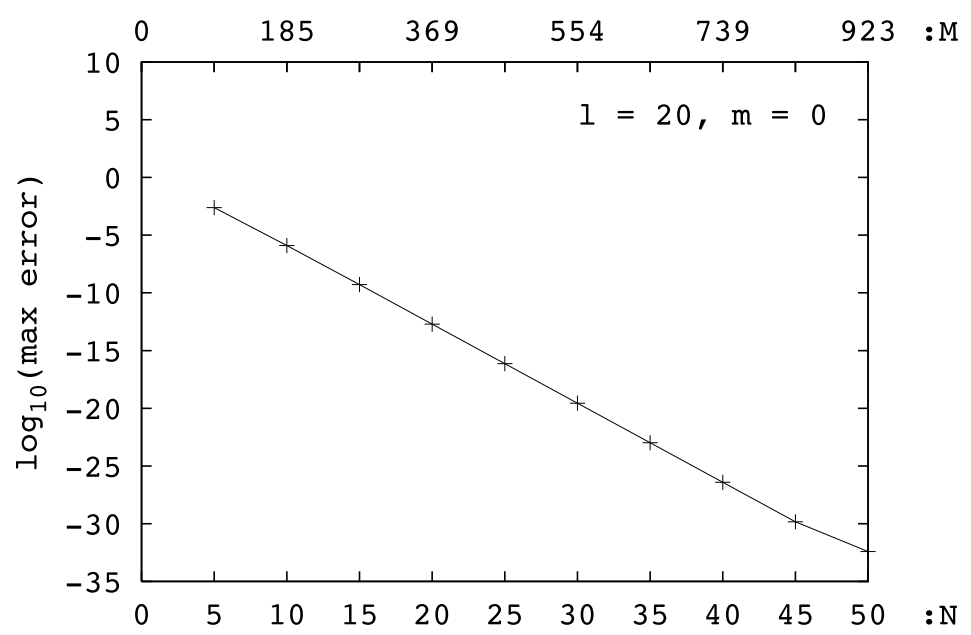

Fig. 3. Errors for $f=\operatorname{sinc}^{20}$ with $m=0$ and $d=\pi^{-1} \log \pi$ 
Table 13. $\log _{10}\left(\max\right.$ error) for $f=\operatorname{sinc}^{l}$ with $d=\pi^{-1} \log \pi$

\begin{tabular}{l||rr|rr|rr}
\multicolumn{1}{l||}{} & \multicolumn{2}{c|}{$m=0$} & \multicolumn{2}{c|}{$m=1$} & \multicolumn{2}{c}{$m=2$} \\
& $N=10$ & $N=40$ & $N=10$ & $N=40$ & $N=10$ & $N=40$ \\
\hline$l=5$ & -6.94 & -27.69 & -5.01 & -25.15 & -2.79 & -22.32 \\
$l=10$ & -6.55 & -27.25 & -4.63 & -24.72 & -2.42 & -21.49 \\
$l=15$ & -6.20 & -26.83 & -4.28 & -24.29 & -2.09 & -21.16 \\
$l=20$ & -5.89 & -26.41 & -3.97 & -23.88 & -1.80 & -20.62
\end{tabular}

Table 14. $\log _{10}$ (max error) $/ N$ for $f=\operatorname{sinc}^{l}$ with $d=\pi^{-1} \log \pi$

\begin{tabular}{l|rrr} 
& $m=0$ & $m=1$ & $m=2$ \\
\hline$l=5$ & -0.690 & -0.673 & -0.656 \\
$l=10$ & -0.689 & -0.672 & -0.643 \\
$l=15$ & -0.688 & -0.670 & -0.644 \\
$l=20$ & -0.685 & -0.668 & -0.638
\end{tabular}

\section{Proofs}

We first note the following fact, the proof of which is omitted.

LEMMA 5.1. For arbitrary nonnegative real numbers $a, b$, and $\tau$, we have $(a+b)^{\tau} \leq 2^{\tau}\left(a^{\tau}+b^{\tau}\right)$, where $0^{0}=1$ by convention.

\subsection{Proof of Lemma 2.1}

For $n \in \mathbf{Z}_{+}$, we set $V_{n}:=\{x+y \mathrm{i}|| x|=(n+1 / 2) h| y \mid, \leq d\}$ and $U_{n}:=$ $\{x+y \mathrm{i}|| x|\leq(n+1 / 2) h| y \mid,=d\}$, where $\mathbf{Z}_{+}$is the set of nonnegative ingeters. By the residue theorem we have

$$
\begin{aligned}
& f(x)-\sum_{k=-n}^{n} f(k h)\left(\frac{\sin (\pi(x-k h) / h)}{\pi(x-k h) / h} \exp \left[-\frac{(x-k h)^{2}}{2 r^{2} h^{2}}\right]\right) \\
& =\frac{1}{2 \pi \mathrm{i}} \int_{V_{n} \cup U_{n}} \frac{\sin (\pi x / h)}{z-x} \exp \left[-\frac{(x-z)^{2}}{2 r^{2} h^{2}}\right] \frac{f(z)}{\sin (\pi z / h)} \mathrm{d} z,
\end{aligned}
$$

and hence it suffices to estimate

$$
\lim _{n \rightarrow \infty} \frac{1}{2 \pi \mathrm{i}} \int_{V_{n} \cup U_{n}} \frac{\mathrm{d}^{m}}{\mathrm{~d} x^{m}}\left(\frac{\sin (\pi x / h)}{z-x} \exp \left[-\frac{(x-z)^{2}}{2 r^{2} h^{2}}\right]\right) \frac{f(z)}{\sin (\pi z / h)} \mathrm{d} z .
$$

Note that

$$
\begin{aligned}
& \frac{\mathrm{d}^{m}}{\mathrm{~d} x^{m}}\left(\frac{\sin (\pi x / h)}{z-x} \exp \left[-\frac{(x-z)^{2}}{2 r^{2} h^{2}}\right]\right) \\
& =\sum_{p=0}^{m} \sum_{l=0}^{p} \frac{m !}{l !(m-p) !} \frac{(-1)^{m-p}}{(\sqrt{2} r h)^{m-p}}\left(\frac{\pi}{h}\right)^{l} \frac{\sin (\pi x / h+\pi l / 2)}{(z-x)^{p-l+1}} \\
& \cdot H_{m-p}\left(\frac{x-z}{\sqrt{2} r h}\right) \exp \left[-\frac{(x-z)^{2}}{2 r^{2} h^{2}}\right]
\end{aligned}
$$


where $H_{j}(x)$ is the $j$-th degree Hermite polynomial

$$
H_{j}(x):=(-1)^{j} \exp \left(x^{2}\right) \frac{\mathrm{d}^{j}}{\mathrm{~d} x^{j}} \exp \left(-x^{2}\right)=\sum_{i=0}^{\lfloor j / 2\rfloor} \frac{(-1)^{i} j !(2 x)^{j-2 i}}{i !(j-2 i) !} .
$$

We assume that $-L \leq x \leq L$ in the following.

Estimate of the integral on $V_{n}$ : By (5.2), it follows from

$$
\lim _{n \rightarrow \infty} \int_{V_{n}} \frac{1}{|z-x|^{p-l+1}}\left|H_{m-p}\left(\frac{x-z}{\sqrt{2} r h}\right)\right|\left|\exp \left[-\frac{(x-z)^{2}}{2 r^{2} h^{2}}\right]\right| \frac{|f(z)|}{|\sin (\pi z / h)|}|\mathrm{d} z|=0
$$

that the integral on $V_{n}$ of (5.1) converges to 0 as $n \rightarrow \infty$. We assume that $n$ is sufficiently large. For $z \in V_{n}$ we have

$$
\begin{aligned}
& (n+1 / 2) h-L \leq|z-x| \leq(n+1 / 2) h+L+d, \\
& \operatorname{Re}(x-z)^{2}=[ \pm(n+1 / 2) h-x]^{2}-(\operatorname{Im} z)^{2} \geq[(n+1 / 2) h-L]^{2}-d^{2}, \\
& |z| \leq(n+1 / 2) h+d, \\
& |\sin (\pi z / h)|=|\cosh (\pi \operatorname{Im} z / h)| \geq 1,
\end{aligned}
$$

and therefore

$$
\begin{aligned}
& \int_{V_{n}} \frac{1}{|z-x|^{p-l+1}}\left|H_{m-p}\left(\frac{x-z}{\sqrt{2} r h}\right)\right|\left|\exp \left[-\frac{(x-z)^{2}}{2 r^{2} h^{2}}\right]\right| \frac{|f(z)|}{|\sin (\pi z / h)|}|\mathrm{d} z| \\
& \leq 4 d \cdot \frac{A+B[(n+1 / 2) h+d]^{\alpha}}{[(n+1 / 2) h-L]^{p-l+1}}\left[\sum_{i=0}^{\lfloor j / 2\rfloor} \frac{2^{j-2 i} j !}{i !(j-2 i) !}\left(\frac{(n+1 / 2) h+L+d}{\sqrt{2} r h}\right)^{j-2 i}\right] \\
& \cdot \exp \left[-\frac{[(n+1 / 2) h-L]^{2}-d^{2}}{2 r^{2} h^{2}}\right] \text {, }
\end{aligned}
$$

where $j=m-p$. This implies (5.4).

Estimate of the integral on $U_{n}$ : By (5.2), we have

$$
\begin{array}{r}
\left|\lim _{n \rightarrow \infty} \frac{1}{2 \pi \mathrm{i}} \int_{U_{n}} \frac{\mathrm{d}^{m}}{\mathrm{~d} x^{m}}\left(\frac{\sin (\pi x / h)}{z-x} \exp \left[-\frac{(x-z)^{2}}{2 r^{2} h^{2}}\right]\right) \frac{f(z)}{\sin (\pi z / h)} \mathrm{d} z\right| \\
\leq \lim _{n \rightarrow \infty} \frac{1}{2 \pi} \int_{U_{n}} \sum_{p=0}^{m} \sum_{l=0}^{p} \frac{m !}{l !(m-p) !}\left(\frac{1}{\sqrt{2} r h}\right)^{m-p}\left(\frac{\pi}{h}\right)^{l} \frac{1}{|z-x|^{p-l+1}} \\
\cdot\left|H_{m-p}\left(\frac{x-z}{\sqrt{2} r h}\right)\right|\left|\exp \left[-\frac{(x-z)^{2}}{2 r^{2} h^{2}}\right]\right| \frac{|f(z)|}{|\sin (\pi z / h)|}|\mathrm{d} z| .
\end{array}
$$

For $z=t \pm d \mathrm{i}(t \in \mathbf{R})$ we have

$$
\frac{1}{|z-x|^{p-l+1}}=\frac{1}{|t-x \pm d \mathrm{i}|^{p-l+1}} \leq \frac{1}{d^{p-l+1}} \leq \max \left\{\frac{1}{d}, \frac{1}{d^{m+1}}\right\} \leq \frac{1}{d}+\frac{1}{d^{m+1}},
$$




$$
\begin{gathered}
\left|\exp \left[-\frac{(x-z)^{2}}{2 r^{2} h^{2}}\right]\right|=\left|\exp \left[-\frac{(x-t)^{2} \mp 2(x-t) d \mathrm{i}-d^{2}}{2 r^{2} h^{2}}\right]\right| \\
=\exp \left[-\frac{(x-t)^{2}}{2 r^{2} h^{2}}\right] \exp \left(\frac{d^{2}}{2 r^{2} h^{2}}\right), \\
|f(z)| \leq A+B|t \pm d \mathrm{i}|^{\alpha} \leq A+B(|t|+d)^{\alpha} \\
\frac{1}{|\sin (\pi z / h)|} \leq \frac{1}{\sinh (\pi d / h)} \leq 4 \exp \left(-\frac{\pi d}{h}\right)
\end{gathered}
$$

where the assumption $h \leq \min \{2 \pi d / \log 2, \pi\}$ is used in the last inequality. These estimates and (5.5) imply that

$$
\begin{aligned}
& \left|\lim _{n \rightarrow \infty} \frac{1}{2 \pi \mathrm{i}} \int_{U_{n}} \frac{\mathrm{d}^{m}}{\mathrm{~d} x^{m}}\left(\frac{\sin (\pi x / h)}{z-x} \exp \left[-\frac{(x-z)^{2}}{2 r^{2} h^{2}}\right]\right) \frac{f(z)}{\sin (\pi z / h)} \mathrm{d} z\right| \\
& \leq \frac{2 m !}{\pi}\left(\frac{1}{d}+\frac{1}{d^{m+1}}\right) \exp \left(-\frac{\pi d}{h}+\frac{d^{2}}{2 r^{2} h^{2}}\right) \\
& \cdot \int_{-\infty}^{\infty} \exp \left[-\frac{(x-t)^{2}}{2 r^{2} h^{2}}\right]\left[A+B(|t|+d)^{\alpha}\right] \\
& \cdot \sum_{p=0}^{m} \sum_{l=0}^{p} \frac{1}{l !(m-p) !}\left(\frac{1}{\sqrt{2} r h}\right)^{m-p}\left(\frac{\pi}{h}\right)^{l} \\
& \left.\leq \frac{2(m+1) !}{\pi}\left(\frac{1}{d}+\frac{1}{d^{m+1}}\right) \exp \left(-\frac{\pi d}{h}+\frac{d^{2}}{2 r^{2} h^{2}}\right)\left(\frac{\pi}{h}\right)^{m} H_{m-p}\left(\frac{x-t-d \mathrm{i}}{\sqrt{2} r h}\right)|+| H_{m-p}\left(\frac{x-t+d \mathrm{i}}{\sqrt{2} r h}\right) \mid\right] \mathrm{d} t \\
& \cdot \int_{-\infty}^{\infty} \exp \left[-\frac{(x-t)^{2}}{2 r^{2} h^{2}}\right]\left[A+B(|t|+d)^{\alpha}\right] \\
& \cdot \sum_{p=0}^{m} \frac{1}{(m-p) !}\left(\frac{1}{\sqrt{2} r h}\right)^{m-p} \\
& \cdot\left[\left|H_{m-p}\left(\frac{x-t-d \mathrm{i}}{\sqrt{2} r h}\right)\right|+\left|H_{m-p}\left(\frac{x-t+d \mathrm{i}}{\sqrt{2} r h}\right)\right|\right] \mathrm{d} t .
\end{aligned}
$$

With $\xi=(x-t) /(r h)$, the integral in (5.6) becomes

$$
\begin{aligned}
\int_{-\infty}^{\infty} \exp \left(-\frac{\xi^{2}}{2}\right)\left[A+B(|x-r h \xi|+d)^{\alpha}\right] \\
\quad \cdot \sum_{j=0}^{m} \frac{1}{j !}\left(\frac{1}{\sqrt{2} r h}\right)^{j}\left[\left|H_{j}\left(\frac{r h \xi-d \mathrm{i}}{\sqrt{2} r h}\right)\right|+\left|H_{j}\left(\frac{r h \xi+d \mathrm{i}}{\sqrt{2} r h}\right)\right|\right] r h \mathrm{~d} \xi .
\end{aligned}
$$

In (5.7), it follows from $-L \leq x \leq L$ that

$$
\begin{aligned}
(|x-r h \xi|+d)^{\alpha} & \leq(|x|+r h|\xi|+d)^{\alpha} \\
& \leq[(L+d)+r h|\xi|]^{\alpha} \leq 2^{\alpha}\left[(L+d)^{\alpha}+(r h)^{\alpha}|\xi|^{\alpha}\right]
\end{aligned}
$$


where the last inequality is due to Lemma 5.1. The Hermite polynomial in (5.7) is evaluated as follows. By (5.3), for $\zeta \in \mathbf{C}$ we have

$$
\left|H_{j}(\zeta)\right| \leq 2^{j} j ! \sum_{i=0}^{\lfloor j / 2\rfloor}|\zeta|^{j-2 i} \leq 2^{j} j ! \frac{j+2}{2} \max \left\{1,|\zeta|^{j}\right\} \leq 2^{j-1} j !(j+2)\left(1+|\zeta|^{j}\right),
$$

and therefore

$$
\begin{aligned}
\sum_{j=0}^{m} \frac{1}{j !}\left(\frac{1}{\sqrt{2} r h}\right)^{j}\left|H_{j}(\zeta)\right| & \leq \sum_{j=0}^{m}\left(\frac{\sqrt{2}}{r h}\right)^{j} \frac{(j+2)}{2}\left(1+|\zeta|^{j}\right) \\
& \leq \frac{(m+2)}{2} \max \left\{1,\left(\frac{\sqrt{2}}{r h}\right)^{m}\right\} \sum_{j=0}^{m}\left(1+|\zeta|^{j}\right) \\
& \leq \frac{(m+2)}{2}\left[1+\left(\frac{\sqrt{2}}{r h}\right)^{m}\right] \sum_{j=0}^{m}\left(1+|\zeta|^{j}\right) \\
& \leq \frac{(m+2)}{2}\left[1+\left(\frac{\sqrt{2}}{r h}\right)^{m}\right](m+1)\left(1+\max \left\{1,|\zeta|^{m}\right\}\right) \\
& \leq \frac{(m+1)(m+2)}{2}\left[1+\left(\frac{\sqrt{2}}{r h}\right)^{m}\right]\left(2+|\zeta|^{m}\right)
\end{aligned}
$$

Substituting $\zeta=(r h \xi \pm d \mathrm{i}) / \sqrt{2} r h$, we obtain

$$
\begin{aligned}
& \sum_{j=0}^{m} \frac{1}{j !}\left(\frac{1}{\sqrt{2} r h}\right)^{j}\left|H_{j}\left(\frac{r h \xi \pm d \mathrm{i}}{\sqrt{2} r h}\right)\right| \\
& \leq \frac{(m+1)(m+2)}{2}\left[1+\left(\frac{\sqrt{2}}{r h}\right)^{m}\right]\left(2+\left|\frac{\xi}{\sqrt{2}} \pm \frac{d \mathrm{i}}{\sqrt{2} r h}\right|^{m}\right) \\
& \leq \frac{(m+1)(m+2)}{2}\left[1+\left(\frac{\sqrt{2}}{r h}\right)^{m}\right]\left[2+\left(\left|\frac{\xi}{\sqrt{2}}\right|+\left|\frac{d}{\sqrt{2} r h}\right|\right)^{m}\right] \\
& \leq \frac{(m+1)(m+2)}{2}\left[1+\left(\frac{\sqrt{2}}{r h}\right)^{m}\right]\left[2+2^{m}\left(\left|\frac{\xi}{\sqrt{2}}\right|^{m}+\left|\frac{d}{\sqrt{2} r h}\right|^{m}\right)\right],
\end{aligned}
$$

where the last inequality is due to Lemma 5.1. Note that this inequality holds good even when $m=0$.

It follows from (5.6), (5.7), (5.8), and (5.9) that

$$
\begin{gathered}
\lim _{n \rightarrow \infty} \frac{1}{2 \pi \mathrm{i}} \int_{U_{n}} \frac{\mathrm{d}^{m}}{\mathrm{~d} x^{m}}\left(\frac{\sin (\pi x / h)}{z-x} \exp \left[-\frac{(x-z)^{2}}{2 r^{2} h^{2}}\right]\right) \frac{f(z)}{\sin (\pi z / h)} \mathrm{d} z \mid \\
\leq \frac{2(m+1) !}{\pi}\left(\frac{1}{d}+\frac{1}{d^{m+1}}\right) \exp \left(-\frac{\pi d}{h}+\frac{d^{2}}{2 r^{2} h^{2}}\right)\left(\frac{\pi}{h}\right)^{m} r h(m+1)(m+2) \\
\cdot\left(1+\left(\frac{\sqrt{2}}{r h}\right)^{m}\right) \int_{-\infty}^{\infty} \exp \left(-\frac{\xi^{2}}{2}\right)\left[A+2^{\alpha} B\left[(L+d)^{\alpha}+(r h)^{\alpha}|\xi|^{\alpha}\right]\right] \\
\cdot\left[2+2^{m}\left(\left|\frac{\xi}{\sqrt{2}}\right|^{m}+\left|\frac{d}{\sqrt{2} r h}\right|^{m}\right)\right] \mathrm{d} \xi
\end{gathered}
$$




$$
\begin{aligned}
& =\frac{m+1}{m+3} C_{0} \exp \left(-\frac{\pi d}{h}+\frac{d^{2}}{2 r^{2} h^{2}}\right) \\
& \cdot \int_{-\infty}^{\infty} \exp \left(-\frac{\xi^{2}}{2}\right)\left[C_{1}+C_{2}|\xi|^{\alpha}\right]\left[C_{3}+2^{m / 2}|\xi|^{m}\right] \mathrm{d} \xi \\
& =\exp \left(-\frac{\pi d}{h}+\frac{d^{2}}{2 r^{2} h^{2}}\right) \cdot C_{0}\left[C_{1} C_{3} \sqrt{2 \pi}+C_{2} C_{3} 2^{\frac{\alpha+1}{2}} \Gamma\left(\frac{\alpha+1}{2}\right)\right. \\
& \left.\quad+C_{1} 2^{\frac{2 m+1}{2}} \Gamma\left(\frac{m+1}{2}\right)+C_{2} 2^{\frac{\alpha+2 m+1}{2}} \Gamma\left(\frac{\alpha+m+1}{2}\right)\right],
\end{aligned}
$$

where $C_{0}, \ldots, C_{3}$ are defined in $(2.3)-(2.6)$, and we used

$$
\int_{-\infty}^{\infty}|\xi|^{p} \exp \left(-\frac{\xi^{2}}{2}\right) \mathrm{d} \xi=2^{\frac{p+1}{2}} \Gamma\left(\frac{p+1}{2}\right) .
$$

\subsection{Proof of Lemma 2.2}

We prepare the following lemma.

Lemma 5.2. Let $p$ be a nonnegative integer, $N$ a positive integer, and $r$ a positive number. If $N \geq \sqrt{p} r$, we have

$$
\sum_{n=N+1}^{\infty} n^{p} \exp \left(-\frac{n^{2}}{2 r^{2}}\right) \leq \frac{(p+1) ! ! \max \left\{N^{p}, r^{p}\right\} r^{2}}{N} \exp \left(-\frac{N^{2}}{2 r^{2}}\right) .
$$

Proof. Put $\eta(x):=x^{p} \exp \left[-x^{2} /\left(2 r^{2}\right)\right]$. Since

$$
\eta^{\prime}(x)=\left(p x^{p-1}-\frac{x^{p+1}}{r^{2}}\right) \exp \left(-\frac{x^{2}}{2 r^{2}}\right)
$$

$\eta(x)$ decreases at $x \geq \sqrt{p} r$. Since $N \geq \sqrt{p} r$ by the assumption, we have an estimate by an integral:

$$
\sum_{n=N+1}^{\infty} n^{p} \exp \left(-\frac{n^{2}}{2 r^{2}}\right) \leq \int_{N}^{\infty} x^{p} \exp \left(-\frac{x^{2}}{2 r^{2}}\right) \mathrm{d} x
$$

Denote by $I_{p}$ the integral of the right hand side in (5.10). By integration by parts, we have

$$
\begin{aligned}
I_{p} & =-r^{2} \int_{N}^{\infty} x^{p-1}\left(-\frac{x}{r^{2}}\right) \exp \left(-\frac{x^{2}}{2 r^{2}}\right) \mathrm{d} x=-r^{2} \int_{N}^{\infty} x^{p-1} \frac{\mathrm{d}}{\mathrm{d} x} \exp \left(-\frac{x^{2}}{2 r^{2}}\right) \mathrm{d} x \\
& =-r^{2}\left[-N^{p-1} \exp \left(-\frac{N^{2}}{2 r^{2}}\right)-(p-1) \int_{N}^{\infty} x^{p-2} \exp \left(-\frac{x^{2}}{2 r^{2}}\right) \mathrm{d} x\right] \\
& =N^{p-1} r^{2} \exp \left(-\frac{N^{2}}{2 r^{2}}\right)+(p-1) r^{2} I_{p-2} .
\end{aligned}
$$


Iterating the similar operation, we obtain

$$
I_{p}=(p-1) ! !\left[\left[\sum_{i=0}^{k-1} \frac{N^{p-2 i-1} r^{2 i}}{(p-2 i-1) ! !}\right] r^{2} \exp \left(-\frac{N^{2}}{2 r^{2}}\right)+\frac{r^{2 k}}{(p-2 k-1) ! !} I_{p-2 k}\right]
$$

for $k=0,1, \ldots,\lfloor p / 2\rfloor$, where we set $(-1) ! !=0 ! !=1$. In the case of $k=\lfloor p / 2\rfloor$, it holds that $I_{p-2 k}=I_{1}$ or $I_{0}$. These integrals are evaluated as follows:

$$
\begin{aligned}
I_{1} & =r^{2} \exp \left(-\frac{N^{2}}{2 r^{2}}\right) \\
I_{0} & =\sqrt{2} r \int_{N /(\sqrt{2} r)}^{\infty} \exp \left(-u^{2}\right) \mathrm{d} u \\
& \leq \sqrt{2} r \cdot \frac{\sqrt{2} r}{2 N} \exp \left(-\frac{N^{2}}{2 r^{2}}\right)=\frac{r^{2}}{N} \exp \left(-\frac{N^{2}}{2 r^{2}}\right)
\end{aligned}
$$

where the latter is due to the general fact that

$$
\int_{t}^{\infty} \exp \left(-u^{2}\right) \mathrm{d} u \leq \frac{\exp \left(-t^{2}\right)}{2 t}
$$

holds for $t>0$. It follows from (5.11), (5.12), and (5.13) that

$$
\begin{aligned}
I_{p} & \leq(p-1) ! !\left[\sum_{i=0}^{\lfloor p / 2\rfloor} \frac{N^{p-2 i-1} r^{2 i}}{(p-2 i-1) ! !}\right] r^{2} \exp \left(-\frac{N^{2}}{2 r^{2}}\right) \\
& \leq N^{p-1}(p-1) ! !\left(\sum_{i=0}^{\lfloor p / 2\rfloor} \frac{r^{2 i}}{N^{2 i}}\right) r^{2} \exp \left(-\frac{N^{2}}{2 r^{2}}\right) \\
& \leq N^{p-1}(p-1) ! !\left(\sum_{i=0}^{\lfloor p / 2\rfloor} \max \left\{1, \frac{r^{p}}{N^{p}}\right\}\right) r^{2} \exp \left(-\frac{N^{2}}{2 r^{2}}\right) \\
& \leq N^{p-1}(p+1) ! ! \max \left\{1, \frac{r^{p}}{N^{p}}\right\} r^{2} \exp \left(-\frac{N^{2}}{2 r^{2}}\right) \\
& =\frac{(p+1) ! ! \max \left\{N^{p}, r^{p}\right\} r^{2}}{N} \exp \left(-\frac{N^{2}}{2 r^{2}}\right) .
\end{aligned}
$$

With this expression and (5.10), we obtain the conclusion.

We now prove Lemma 2.2. In a similar manner to (5.2), we see

$$
\begin{aligned}
& \frac{\mathrm{d}^{m}}{\mathrm{~d} x^{m}}\left(\frac{\sin (\pi(x-k h) / h)}{\pi(x-k h) / h} \exp \left[-\frac{(x-k h)^{2}}{2 r^{2} h^{2}}\right]\right) \\
& =\sum_{p=0}^{m} \sum_{l=0}^{p} \frac{m !}{l !(m-p) !} \frac{(-1)^{m-l}}{(\sqrt{2} r h)^{m-p}}\left(\frac{\pi}{h}\right)^{l-1} \frac{\sin (\pi(x-k h) / h+\pi l / 2)}{(x-k h)^{p-l+1}} \\
& \cdot H_{m-p}\left(\frac{x-k h}{\sqrt{2} r h}\right) \exp \left[-\frac{(x-k h)^{2}}{2 r^{2} h^{2}}\right],
\end{aligned}
$$


and therefore

$$
\begin{gathered}
\left|\left(\mathcal{G}_{h}^{(m)} f\right)(x)-\left(\mathcal{T}_{N, h}^{(m)} f\right)(x)\right| \\
\leq \sum_{\substack{k-\lceil x / h\rceil>N \\
k-\lfloor x / h\rfloor<-N}}|f(k h)| \sum_{p=0}^{m} \sum_{l=0}^{p} \frac{m !}{l !(m-p) !} \frac{1}{(\sqrt{2} r h)^{m-p}}\left(\frac{\pi}{h}\right)^{l-1} \frac{1}{h^{p-l+1}|x / h-k|^{p-l+1}} \\
\cdot\left|H_{m-p}\left(\frac{x / h-k}{\sqrt{2} r}\right)\right| \exp \left[-\frac{(x / h-k)^{2}}{2 r^{2}}\right]
\end{gathered}
$$

We assume that $-L \leq x \leq L$.

We estimate the sum in (5.14) in the case of $k-\lceil x / h\rceil>N$. Note that

$$
N<k-\left\lceil\frac{x}{h}\right\rceil \leq k-\frac{x}{h} \leq k-\left\lceil\frac{x}{h}\right\rceil+1 .
$$

By (5.3), setting

$$
a_{i}(t):=\frac{j !(2 t)^{j-2 i}}{i !(j-2 i) !}
$$

for $t \in \mathbf{R}$, we have that

$$
H_{j}(t)=\sum_{i=0}^{\lfloor j / 2\rfloor}(-1)^{i} a_{i}(t),
$$

and that $a_{i}(t)(i=0,1, \ldots,\lfloor j / 2\rfloor)$ have the same sign. Furthermore, if $|t| \geq j / 2$, we have $\left|a_{0}(t)\right| \geq \cdots \geq\left|a_{\lfloor j / 2\rfloor}(t)\right|$, and therefore $\left|H_{j}(t)\right| \leq\left|a_{0}(t)\right|$.

Since $k-x / h>N \geq m r / \sqrt{2}$ holds by the assumption, setting $t=(k-$ $x / h) /(\sqrt{2} r)$ in the above discussion, we have

$$
\left|H_{j}\left(\frac{x / h-k}{\sqrt{2} r}\right)\right| \leq\left(\frac{\sqrt{2}|x / h-k|}{r}\right)^{j}=\left[\frac{\sqrt{2}(k-x / h)}{r}\right]^{j}
$$

for $j=0,1, \ldots, m$.

By (5.15) and (5.16), we have

$$
\begin{gathered}
\sum_{k-\lceil x / h\rceil>N}|f(k h)| \sum_{p=0}^{m} \sum_{l=0}^{p} \frac{m !}{l !(m-p) !} \frac{1}{(\sqrt{2} r h)^{m-p}}\left(\frac{\pi}{h}\right)^{l-1} \frac{1}{h^{p-l+1}|x / h-k|^{p-l+1}} \\
\cdot\left|H_{m-p}\left(\frac{x / h-k}{\sqrt{2} r}\right)\right| \exp \left[-\frac{(x / h-k)^{2}}{2 r^{2}}\right] \\
\leq \frac{1}{N h^{m}} \sum_{k-\lceil x / h\rceil>N}\left[A+B\left[\left(k-\left\lceil\frac{x}{h}\right\rceil\right) h+(L+h)\right]^{\alpha}\right] \\
\cdot \sum_{p=0}^{m} \sum_{l=0}^{p} \frac{m ! \pi^{l-1}}{l !(m-p) !}\left(\frac{k-\lceil x / h\rceil+1}{r^{2}}\right)^{m-p} \exp \left[-\frac{(k-\lceil x / h\rceil)^{2}}{2 r^{2}}\right]
\end{gathered}
$$




$$
\begin{aligned}
\leq \frac{1}{N h^{m}} \sum_{n=N+1}^{\infty}\left[A+B[n h+(L+h)]^{\alpha}\right] \\
\qquad \sum_{p=0}^{m} \sum_{l=0}^{p} \frac{m ! \pi^{l-1}}{l !(m-p) !}\left(\frac{n+1}{r^{2}}\right)^{m-p} \exp \left(-\frac{n^{2}}{2 r^{2}}\right) .
\end{aligned}
$$

Furthermore, noting that

$$
\begin{aligned}
& {[n h+(L+h)]^{\alpha} } \leq 2^{\alpha}\left[(n h)^{\alpha}+(L+h)^{\alpha}\right] \\
& \leq 2^{\alpha}\left[(L+h)^{\alpha}+2^{\alpha} h^{\alpha}\right]+2^{2 \alpha} h^{\alpha}(n-1)^{\alpha}, \\
& \sum_{l=0}^{p} \frac{\pi^{l-1}}{l !} \leq \frac{1}{\pi} \sum_{l=0}^{\infty} \frac{\pi^{l}}{l !}=\frac{\mathrm{e}^{\pi}}{\pi}, \\
& \sum_{p=0}^{m} \frac{1}{(m-p) !}\left(\frac{n+1}{r^{2}}\right)^{m-p} \leq \exp \left(\frac{n+1}{r^{2}}\right),
\end{aligned}
$$

where Lemma 5.1 is used, we have

$$
\begin{aligned}
& \frac{1}{N h^{m}} \sum_{n=N+1}^{\infty}\left[A+B[n h+(L+h)]^{\alpha}\right] \sum_{p=0}^{m} \sum_{l=0}^{p} \frac{m ! \pi^{l-1}}{l !(m-p) !}\left(\frac{n+1}{r^{2}}\right)^{m-p} \exp \left(-\frac{n^{2}}{2 r^{2}}\right) \\
& \leq \frac{m ! \mathrm{e}^{\pi}}{N h^{m} \pi} \sum_{n=N+1}^{\infty}\left[A+2^{\alpha} B\left[(L+h)^{\alpha}+2^{\alpha} h^{\alpha}\right]+2^{2 \alpha} B h^{\alpha}(n-1)^{\alpha}\right] \\
& \cdot \exp \left(\frac{n+1}{r^{2}}\right) \exp \left(-\frac{n^{2}}{2 r^{2}}\right) \\
& \leq \frac{m ! \mathrm{e}^{\pi} \mathrm{e}^{\frac{3}{2 r^{2}}}}{N h^{m} \pi} \sum_{n=N+1}^{\infty}\left[A+2^{\alpha} B\left[(L+h)^{\alpha}+2^{\alpha} h^{\alpha}\right]+2^{2 \alpha} B h^{\alpha}(n-1)^{\alpha}\right] \\
& \cdot \exp \left[-\frac{(n-1)^{2}}{2 r^{2}}\right] .
\end{aligned}
$$

Applying Lemma 5.2 (by the assumption $N \geq \sqrt{\lceil\alpha\rceil} r+1$, we have only to replace $N$ with $N-1$ and set $p=0,\lceil\alpha\rceil$ ), we have

$$
\begin{aligned}
& \frac{m ! \mathrm{e}^{\pi} \mathrm{e}^{\frac{3}{2 r^{2}}}}{N h^{m} \pi} \sum_{n=N+1}^{\infty}\left[A+2^{\alpha} B\left[(L+h)^{\alpha}+2^{\alpha} h^{\alpha}\right]+2^{2 \alpha} B h^{\alpha}(n-1)^{\alpha}\right] \exp \left[-\frac{(n-1)^{2}}{2 r^{2}}\right] \\
& \leq \frac{m ! \mathrm{e}^{\pi} \mathrm{e}^{\frac{3}{2 r^{2}}} r^{2}}{N(N-1) h^{m} \pi}\left(C_{1}^{\prime}+C_{2}^{\prime}\right) \exp \left[-\frac{(N-1)^{2}}{2 r^{2}}\right]
\end{aligned}
$$

where $C_{1}^{\prime}$ and $C_{2}^{\prime}$ are defined in $(2.8)$ and (2.9), respectively. 
Noting that the sum in (5.14) in the case of $k-\lfloor x / h\rfloor<-N$ can be estimated in a similar manner, we see from $(5.14),(5.17),(5.18)$, and (5.19) that

$$
\left|\left(\mathcal{G}_{h}^{(m)} f\right)(x)-\left(\mathcal{T}_{N, h}^{(m)} f\right)(x)\right| \leq C_{0}^{\prime}\left(C_{1}^{\prime}+C_{2}^{\prime}\right) \exp \left[-\frac{(N-1)^{2}}{2 r^{2}}\right],
$$

where $C_{0}^{\prime}$ is defined in (2.7). Thus we have proven Lemma 2.2.

Acknowledgements. The authors are thankful to to the anonymous referees for valuable suggestions. This work is supported by the 21st Century COE Program on Information Science and Technology Strategic Core and a Grant-in-Aid of the Ministry of Education, Culture, Sports, Science and Technology of Japan. The first author is supported by the Research Fellowship of the Japan Society for the Promotion of Science for Young Scientists.

\section{References}

[1] J.R. Higgins, Sampling Theory in Fourier and Signal Analysis: Foundations. Clarendon Press, Oxford, 1996.

[ 2 ] L.W. Qian, On the regularized Whittaker-Kotel'nikov-Shannon sampling formula. Proc. Amer. Math. Soc., 131 (2003), 1169-1176.

[ 3 ] L.W. Qian and D.B. Creamer, Localization of the generalized sampling series and its numerical application. SIAM J. Numer. Anal., 43 (2006), 2500-2516.

[ 4 ] L.W. Qian and D.B. Creamer, A modification of the sampling series with a Gaussian multiplier. Sampling Theory in Signal and Image Processing, 5 (2006), 1-19.

[ 5 ] L.W. Qian and H. Ogawa, Modified sinc kernels for the localized sampling series. Sampling Theory in Signal and Image Processing, 4 (2005), 121-139.

[6] F. Stenger, Numerical Methods Based on Sinc and Analytic Functions. Springer-Verlag, New York, 1993.

[ 7 ] F. Stenger, Summary of sinc numerical methods. J. Comp. Appl. Math., 121 (2000), 379-420.

[ 8 ] M. Sugihara and T. Matsuo, Recent developments of the sinc numerical methods. J. Comput. Appl. Math., 164-165 (2004), 673-689.

[ 9 ] H. Takahasi, Complex function theory and numerical analysis (in Japanese). Numerical Analysis and Computers, Kokyuroku, RIMS, Kyoto Univ., 253 (1975), 24-37. English translation: Publ. RIMS, Kyoto Univ., 41 (2005), 979-988.

[10] K. Tanaka, M. Sugihara and K. Murota, Error estimate of a Gaussian kernel sampling formula via complex analysis (in Japanese). Trans. Japan Soc. Indust. Appl. Math., 17 (2007), 73-95.

[11] D.C. Wan, B.S.V. Patnaik and G.W. Wei, Discrete singular convolution-finite subdomain method for the solution of incompressible viscous flows. J. Comput. Phys., 180 (2002), 229-255.

[12] G.W. Wei, A unified approach for the solution of the Fokker-Planck equation. J. Phys. A, Mathematical and General, 33 (2000), 4935-4953.

[13] G.W. Wei, Discrete singular convolution for the sine-Gordon equation. Physica D, 137 (2000), 247-259.

[14] S. Zhao and G.W. Wei, Option valuation by using discrete singular convolution. Appl. Math. Comput., 167 (2005), 383-418.

[15] Y.B. Zhao, G.W. Wei and Y. Xiang, Discrete singular convolution for the prediction of high frequency vibration of plates. Int. J. Solids and Struct., 39 (2002), 65-88. 
